\title{
Contribution à l'étude du polyéthisme chez les bourdons, Bombus Latr. (Hymenoptera, Apidae)
}

\section{A. Pouvreau}

Laboratoire de neurobiologie comparée des invertébrés, INRA, CNRS (UA 1190), 91440 Bures-surYvette, France

(reçu le 4 juillet 1988, accepté le 23 février 1989)

Résumé - Les ouvrières de bourdons accomplissent une série ontogénique de tâches durarit leur vie. II y a progression des tâches de nourrices vers celles de butineuses à mesure que les ouvrières vieillissent, mais la relation entre la nature du travail exécuté par une ouvrière et son âge est moins stricte que chez l'abeille domestique. Le polymorphisme des ouvrières de Bombus, plus ou moins marqué selon les espèces, nous a conduit à considérer les relations entre la taille des ouvrières et les fonctions qu'elles assument, ainsi que la répartition des tâches parmi les butineuses.

Bombus — polyethisme - åge — taille — polymorphisme

Summary - Contribution to the study of polyethism in Bumblebees, Bombus Latr. (Hymenoptera, Apidae). The first purpose of our work was to establish a relationship between the changeover from one social function to another and the age of workers in Bombus lucorum L., Pyrobombus (Melanobombus) lapidarius $L$. and Megabombus (Thoracobombus) pascuorum Scop. colonies. We also considered the relationship between the size of workers and the division of labour in species which exhibit a more or less marked polymorphism.

Initially, all the workers are nurses. Some remain so throughout their life, while others change speciality and become foragers. The mean age from which most workers effect their first departure takes place on the 7th or 8th day in B. lucorum and P. lapidarius. Most of the first departures in M. pascuorum workers take place on the 6th day for the bigger individuals, and on the 13th or 14th day for the smaller ones. The modification of behaviour is achieved earlier in big workers than in small ones.

The interchangeability of tasks in the case of experimental removal of workers, as reported by some authors, as well as the development of hypopharyngeal glands, are discussed here.

The size of workers is involved in the labour specialization in bumblebees. Size plays a greater part in the distinction of activities between nurses and foragers in $\mathrm{M}$. pascuorum than in $\mathrm{B}$. lucorum and P. lapidarius (Fig. 1). Polymorphism is more marked in the first species than in the two other ones. Larger workers tend to gather relatively more food than the small ones (Fig. 3). Among the foragers, the small size workers generally gather nectar rather than pollen, the process being reversed in the big individuals (Table III). The division of labour in the sub-caste of foragers is discussed.

Bombus - polyethism - age — body size - polymorphism 
Zusammenfassung - Beltrag zum Studium des Polyethismus bei Hummeln (Hymenopterae, Apoidea, Bombinae, Bombus Latr.). Das erste Ziel unserer Arbeit war die Untersuchung der Beziehung zwischen dem Wechsel von einer sozialen Funktion zur anderen und dem Alter der Arbeiterin in Völkem von Bombus lucorum L., Pyrobombus (Melanobombus) lapidarius L. und Megabombus (Thoracobombus) pascuorum Scop.

Anschließend untersuchten wir die Beziehung zwischen der GröBe der Arbeiterin und der Aufteilung der Arbeit in Arten, die einen mehr oder weniger ausgeprägten Polymorphismus besitzen.

Alle Arbeiterinnen sind am Anfang Ammen. Einige bleiben dabei während ihres ganzen Lebens, andere wechseln ihre Aufgabe und werden Sammlerinnen.

Das durchschnittliche Alter, in dem die meisten Arbeiterinnen ihren ersten Ausflug machen, beträgt bei B. lucorum und $\mathrm{P}$. lapidarius 7 bis 8 Tage (Abb. 1). Bei M. pascuorum findet der erste Ausflug der größeren Individuen am 6. Tag statt, der der kleineren Arbeiterinnen am 13. bis 14. Tag (Abb. 3). Die Änderung des Verhaltens wird also bei den großen Arbeiterinnen früher erreicht als bei den Keinen.

Die Auswechselbarkeit der Aufgaben im Falle einer experimentellen Entnahme von Arbeiterinnen, wie sie von einigen Autoren beschrieben wird, und die Entwicklung der Hypopharynxdrüsen werden hier diskutiert.

Die GröBe der Arbeiterinnen spielt eine Rolle bei der Arbeitsteilung der Hummeln. Der EinfluB der Größe auf die Aufteilung der Aktivitäten von Ammen und Sammlerinnen ist bei der Art M. pascuorum gröBer als bei B. lucorum und P. lapidarius. Der Polymorphismus ist in der ersten Art ausgeprägter als bei den beiden anderen.

Die größeren Arbeiterinnen neigen dazu, größere Mengen Futter zu sammeln als die kleineren. Unter den Sammlerinnen sammeln die kleineren Arbeiterinnen generell eher Nektar als Pollen, bei den großen Individuen ist es umgekehrt (Tabelle III). Die Arbeitsteilung in der Subkaste der Sammlerinnen wird diskutiert.

\section{Bombus - Polyethismus - Alter - Körpergrösse - Polymorphismus}

\section{Introduction}

Les colonies d'insectes sociaux présentent de telles interactions physiologiques, de tels comportements et de telles régulations qu'on ne peut douter d'une intégration des caractéristiques des individus appartenant à une colonie. L'équilibre du groupe social se maintient par un grand nombre de régulations.

La répartition du travail est, selon Michener (1974), l'un des aspects structuraux du polymorphisme social chez les insectes. Pratiquement, toute l'évolution de la vie sociale chez les insectes repose sur l'acquisition du polymorphisme social.
La répartition des tâches au sein de la colonie est fonction de trois composantes (Wilson, 1971). Les deux premières correspondent au polyéthisme de caste et au polyéthisme d'âge, la troisième est soumise à une grande variabilité et subit l'influence de facteurs tels que la taille et l'âge de la colonie, l'alimentation des individus, l'expérience individuelle. Chez les insectes sociaux, une distinction peut être faite entre le polyéthisme * de caste, dans lequel les castes morphologiques sont spécialisées pour répondre à différentes fonctions, et le polyéthisme d'âge, dans lequel un même individu assure différentes spécialisations au cours de son évolution imaginale.

\footnotetext{
* Le terme de polyéthisme a été employé par Weir (1958), en synonymie avec la division du travail, pour qualifier la progression du comportement en fonction de l'âge. Puis Wilson a proposé d'élargir l'utilisation du terme de polyéthisme à l'ensemble de la division du travail.
} 
L'idée d'une répartition du travail en fonction de l'âge est venue de l'observation du polyéthisme chez les abeilles domestiques et remonte au siècle dernier. On en connaît le schéma donné par Rösch (1930), confirmé et précisé depuis par différents auteurs (revue récente par Nowogrodzki, 1984). Chez Apis mellifera L., ces travaux se résument à la distinction de 2 périodes successives : l'une où l'abeille travaille à l'intérieur de la ruche, l'autre où elle n'accomplit que des travaux de récolte.

Les diverses approches utilisées pour expliquer les relations entre la taille et la fonction ou entre le travail exécuté et l'âge de l'insecte ne permettent pas encore de définir clairement le polymorphisme social chez les bourdons. L'utilisation d'une méthode plus fine, par marquage individuel dès l'apparition de l'adulte, pendant toute la période d'activité des ouvrières, suivant une étude comparative, doit permettre d'avoir une meilleure compréhension de ces phénomènes.

Nous avons cherché à mettre en relation le passage d'une fonction sociale à une autre et l'âge des ouvrières de la colonie chez 3 espèces de bourdons : Bombus lucorum L., Pyrobombus (Melanobombus) lapidarius L. et Megabombus (Thoracobombus) pascuorum Scop.

Nous avons été conduit à examiner la relation entre la taille des ouvrières et la répartition des tâches chez des espèces qui présentent un polymorphisme plus ou moins accentué.

\section{Conditions expérimentales}

\section{Matériel biologique}

Les observations sur la répartition du travail ont été effectuées sur les espèces citées précédemment.
Après fondation des nids en serre d'élevage, suivant une technique mise au point au laboratoire (Pouvreau et Marilleau, 1979), les caisses de nidification sont transférées dans la nature, sous un appentis, dès l'apparition des premières ouvrières.

\section{Techniques}

Les observations sont faites soit directement dans le nid afin de noter le comportement des ouvrières, soit à l'extérieur de la caisse d'élevage, dans le but de préciser certaines séquences de l'activité de récolte des bourdons.

Dès l'apparition des adultes, tous les insectes de la colonie sont marqués, après un bref séjour à la température de $5^{\circ} \mathrm{C}$, à l'aide de pastilles numérotées de couleurs différentes, de manière à pouvoir les repérer au cours de leurs diverses activités.

Le repérage des ouvrières butineuses hors du nid s'effectue lorsque celles-ci sortent ou rentrent dans la caisse d'élevage par l'intermediaire d'un sas transparent. Ce dispositif (25 $x$ $6 \times 4 \mathrm{~cm}$ ), construit au moyen d'un assemblage de plaques d'altuglas, comporte une cloison longitudinale qui divise le sas en 2 couloirs, correspondant chacun, soit aux sorties, soit aux rentrées des butineuses. Au voisinage de l'extrémité de ces couloirs, l'interposition de 2 plaques d'altuglas amovibles permet d'interrompre momentanément la sortie ou la rentrée des butineuses, juste le temps de noter leur numéro et la nature de l'aliment récolté.

Pour déterminer l'importance de la taille des ouvrières dans le polyéthisme, nous avons effectué des mesures de celle-ci en prenant comme critère la longueur de la cellule radiale des ailes antérieures. Ce paramètre reflète fidèlement les différences de taille entre les individus (Morse, 1977) et s'applique, après une légère anesthésie par le froid, à des bourdons vivants.

\section{Méthodologie}

Nos observations ont porté sur l'activité des ouvrières pendant toute la période où elles apparaissent dans la colonie. La méthode de notations s'appuie sur l'observation focalisée des ouvrières, pendant une durée d'une heure, à raison de 4 relevés de situation par jour 
effectués à heures fixes $(7 \mathrm{~h}, 9 \mathrm{~h}, 12 \mathrm{~h}, 15 \mathrm{~h}$ GMT). Cette méthode a permis d'obtenir un nombre d'informations suffisant sur le type d'activités des butineuses, et de décrire avec précision les séquences comportementales successives, en rapport avec les soins au couvain et l'approvisionnement de la colonie. Nos observations ont été complétées par un certain nombre de relevés ponctuels (scan sampling; Lenoir, 1979).

Les ouvrières nouvellement apparues peuvent être considérées comme un groupe de base sans différenciation aussi longtemps qu'une spécialisation n'est pas intervenue dans les jours qui suivent.

Les ouvrières de chaque colonie sont ensuite réparties en 3 catégories :

- les «nourrices constantes" regroupent à la fois les ouvrières qui ne quittent jamais leur nid et celles qui consacrent au moins $75 \%$ de leur temps en activités au sein du nid;

- les abutineuses constantes": lorsque les ouvrières passent la plus grande partie de leur temps, à partir de leur première sortie du nid, à récolter de la nourriture (nectar et/ou pollen). Dans le cadre de notre expérimentation, les ouvrières qui manifestent une activité de butinage durant les 4 observations au cours d'une même journée, et pendant une période continue de 3 à 4 semaines, correspondant normalement à leur durée de vie minimale, sont considérées comme des abutineuses constantes";

- les "ouvrières inconstantes": lorsque, au cours de leur vie, elles passent d'une tâche à une autre. Ce changement d'activités peut s'effectuer au cours d'une même journée, ou bien d'un jour à l'autre.

Les colonies de bourdons, du moins celles qui appartiennent à des espéces dont la population d'ouvrières est importante ( $P$. hypnorum, $B$. lucorum, $B$. terrestris, $P$. lapidarius), comportent des gardiennes. Celles-ci sont en très petit nombre et se recrutent aussi bien parmi les butineuses que parmi les nourrices.

Les observations demandent un «suivi» de chaque individu, et les désignations de «nourrice constante», de abutineuse constante», etc., ne peuvent être attribuées qu'a la fin de lá période d'observation des colonies ou à un stade d'évolution avancé.

\section{Expression des résultats}

La comparaison de certains résultats est effectuée à l'aide des moyennes accompagnées de l'écart-type.
L'interprétation statistique est poursuivie au moyen de l'analyse de variance (test F) ou de l'analyse de covariance (régression). Dans ce dernier cas, nous avons calculé l'écart-type du coefficient de régression et le test de signification $t$.

\section{Résultats}

Répartition ontogénique du travail chez les ouvrières de bourdons

La question qui se pose est de savoir dans quelle mesure le changement de comportement des ouvrières peut être affecté par l'âge.

Les résultats (Fig. 1) montrent que, sur l'ensemble des couvées, les premières sorties d'ouvrières s'échelonnent entre le $2^{e}$ ou le $3^{\theta}$ jour et du $14^{\theta}$ au $17^{e}$ jour de leur vie imaginale chez les 3 espéces observées. Le plus grand nombre de premières sorties se situent entre le $7^{\theta}$ et le $10^{\ominus}$ jour chez Bombus lucorum, entre le $5^{\ominus}$ et le $9^{\theta}$ jour chez Pyr. (Mel.) lapidarius, entre le $5^{\ominus}$ et le $14^{\theta}$ jour chez $\mathrm{Meg}$. (Thor.) pascuorum. Ces sorties atteignent leur valeur maximale le $8^{\circ}$ ou le $9^{\theta}$ jour chez $B$. lucorum, et le $7^{\theta}$ jour chez $P$. lapidarius, pour chacune des colonies observées; chez $M$. pascuorum, les résultats montrent un certain nivellement des valeurs avec, cependant, 2 points culminants, apparaissant à des dates voisines : le premier point apparaît au $6^{e}$ jour pour les 3 colonies, le second correspond aux $13^{\theta}$ et $14^{\theta}$ jours, respectivement pour les colonies 27,10 et 48 .

Le calcul des moyennes de l'âge à la première sortie a donné les résultats suivants :

- chez $P$. (M.) lapidarius : $7,24 \pm 2,86 \mathrm{j}$ (col. 81); 7,15 $\pm 2,68$ j (col. 43); 7,24 \pm 2,61 j (col. 54); 
- chez B. lucorum : 8,95 $\pm 3,16 \mathrm{j}$ (col. 63); $8,93 \pm 3,03$ j (col. 40); $9,44 \pm 3,10$ j (col. 87);

- chez M. (T.) pascuorum : 8,72 $\pm 3,68 \mathrm{j}$ (col. 48); $8,89 \pm 3,70$ j (col. 27); 9,92 \pm $3,89 \mathrm{j}$ (col. 10).

La comparaison des moyennes (test $F$ ) entre les colonies d'une espèce ou d'espèces différentes ne montre aucune différence significative.

Afin de préciser le changement de séquences d'activité en liaison avec l'âge, nous avons calculé, pour chaque couvée d'ouvrières, l'âge moyen auquel chaque individu effectuait sa première sortie. L'examen de la Figure 2 permet de constater que les premières sorties d'ouvrières s'effectuent de plus en plus précocement au fur et à mesure que les couvées se succèdent. La droite de régression (dont le coefficient de régression est négatif), tracée pour chaque colonie, met en évidence la diminution de l'âge moyen des ouvrières à leur première sortie en fonction du numéro de la couvée.
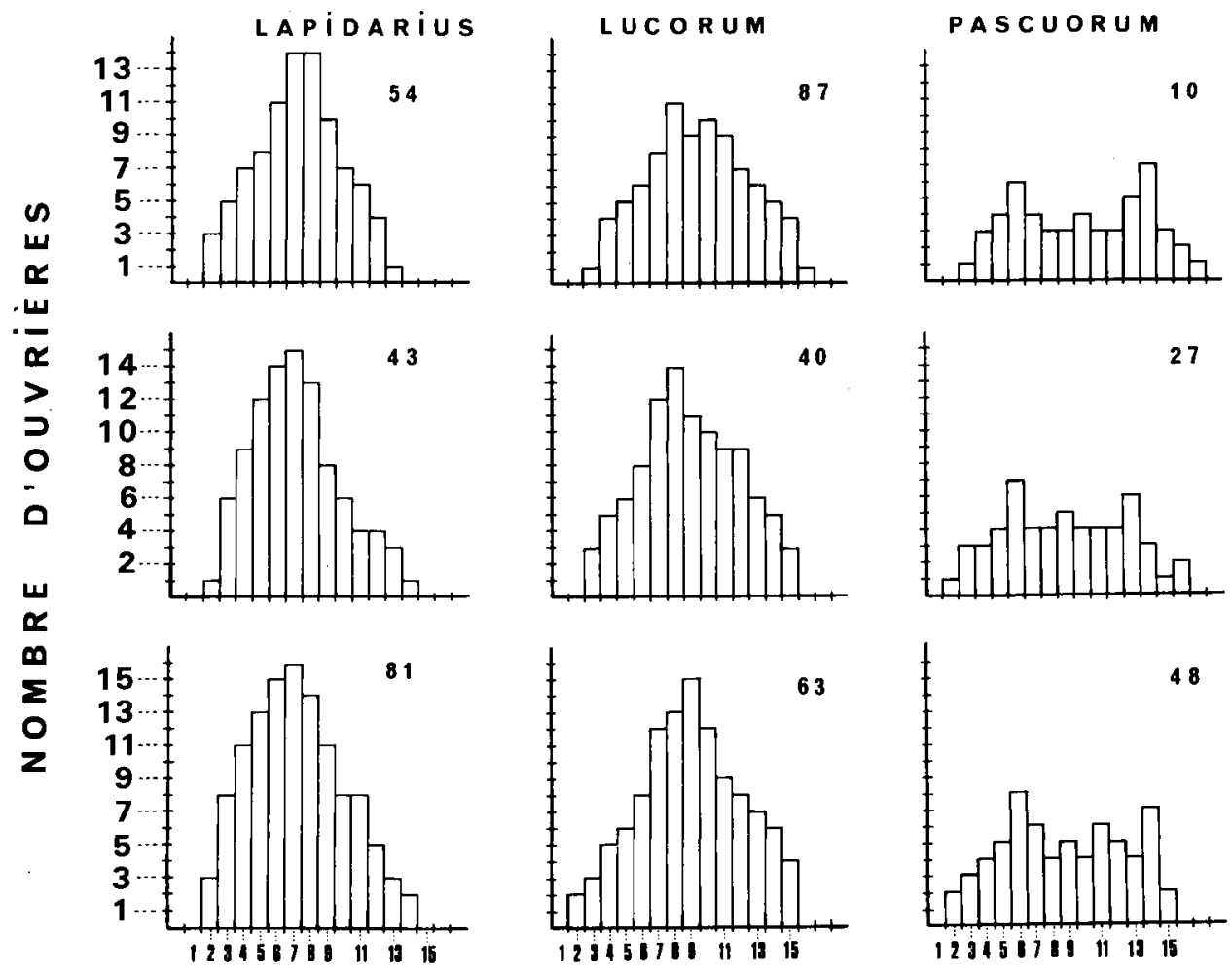

AGE (EN JOURS)

Fig. 1. Nombres d'ouvrières de bourdons sortant du nid pour la première fois en fonction de leur âge. En abscisses : âge (en jour) des ouvrières à leur première sortie. En ordonnées : nombres d'ouvrières sortant du nid pour la premiere fois. Pyr. (Mel.) lapidarius (colonies 81, 43, 54); B. lucorum (colonies 63, 40, 87); Meg. (Thor.) pascuorum (colonies 48, 27, 10). 
Taille des ouvrières et polyéthisme

L'observation d'une colonie de bourdons permet généralement de distinguer des différences plus ou moins sensibles dans la taille des ouvrières (polymorphisme).

Nous avons cherché à établir des relations entre la taille des ouvrières de bourdons et, d'une part, la nature des tâches exécutées au cours de leur vie, d'autre part, l'âge auquel les ouvrières effectuent leur première sortie.
L'examen du Tableau I permet de remarquer :

- l'existence, chez les 3 espèces de Bombus étudiées, d'un gradient de taille en fonction de l'appartenance à l'une des sous-castes et qui s'exprime dans l'ordre décroissant suivant : butineuses constantes > ouvrières inconstantes > nourrices constantes;

- des différences entre les moyennes de la longueur de la cellule radiale plus accusées chez $M$. pascuorum que chez les 2
P. I apidarius
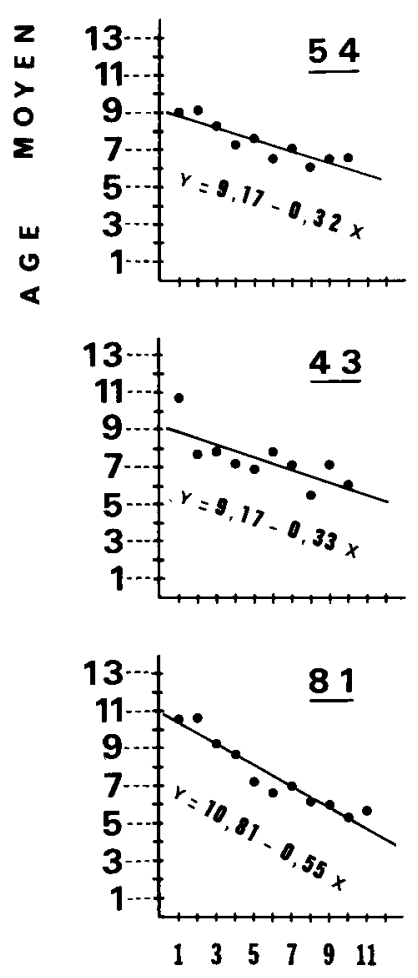

B. I ucorum
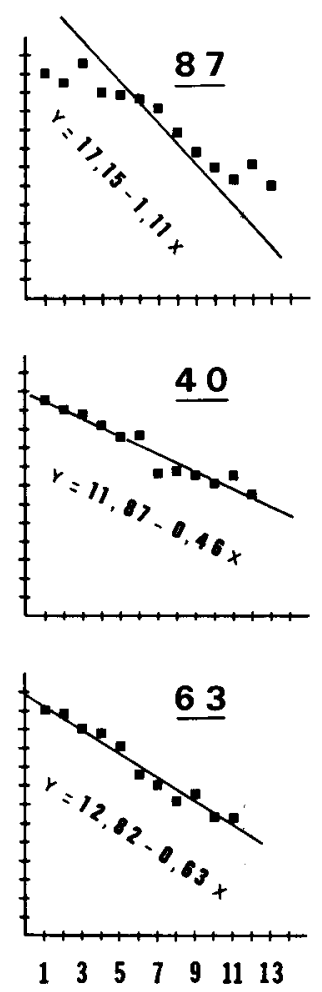

M. pascuorum
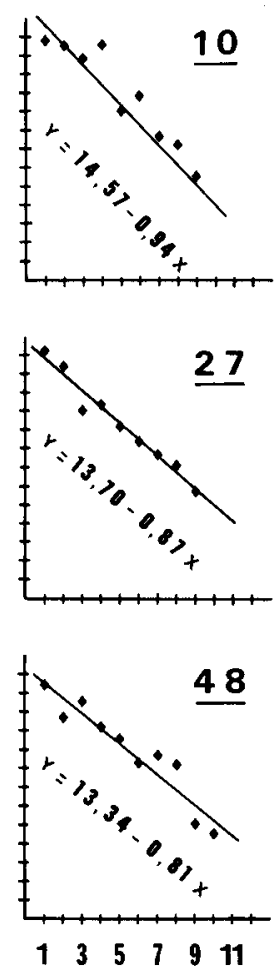

NUMÉRO DE LA COUVÉE

Fig. 2. Age moyen des ouvrières de bourdons, chez trois espèces, à leur première sortie en fonction du numéro de la couvée. En abscisses : numéro de la couvée. En ordonnées : âge moyen (en jours) des ouvrières à leur première sortie. Pyr. (Mel.) lapidarius (colonies 81, 43, 54); B. lucorum (colonies 63, 40, 87); Meg. (Thor.) pascuorum (colonies 48, 27, 10). 


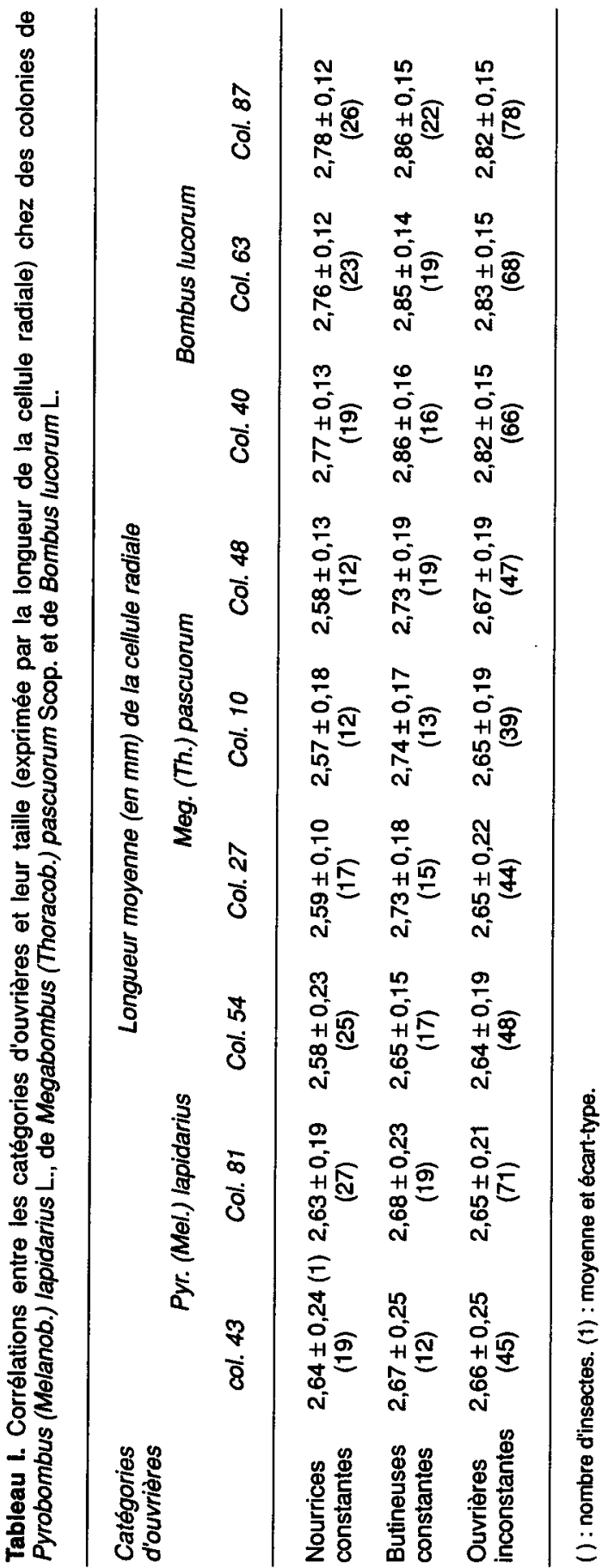


autres espèces, en fonction de la souscaste.

Le test de signification $F$ de comparaison entre les moyennes ne révèle aucune différence significative entre les valeurs extrêmes, c'est-à-dire entre celles qui correspondent aux «nourrices constantes» et celles qui correspondent aux «butineuses constantes", chez $P$. (M.) lapidarius et $B$. lucorum. La différence est significative (F $>5 \%$ ) chez $M$. (T.) pascuorum.

Dans l'étude des relations entre la taille d'une ouvrière et son âge lors de sa première sortie, nous avons considéré les ouvrières appartenant aux catégories des "butineuses constantes" et des "ouvrières inconstantes», autrement dit celles qui ont été observées sortant du nid pour faire des provisions.

Les ouvrières sont réparties en 4 groupes correspondant chacun à un intervalle de taille défini par les longueurs de la cellule radiale.

Les résultats sont rapportés sur la Figure 3, où il apparaît que, d'une manière générale, les ouvrières de grande taille effectuent leur première sortie à un âge plus précoce que les petites ouvrières.

Les différences entre les intervalles de taille sont plus importantes chez $M$. pascuorum que chez $B$. lucorum et chez $P$. lapidarius.

Des analyses de variance mettent ce phénomène en relief. L'examen du Tableau II montrent que chez $P$. lapidarius les différences entre les groupes correspondant aux intervalles de taille ne sont pas significatives, à l'exception de 3 comparaisons. Chez B. lucorum, une colonie présente des différences non significatives, tandis que dans les 2 autres les différences apparaissent plus nettes au niveau des groupes les plus éloignés l'un de l'autre (A-C; A-D). Chez pascuorum, les différences sont pour la plupart hautement significatives.
Relations entre la taille des butineuses et la nature des aliments récoltés

Nous avons cherché s'il existait une relation entre la taille des ouvrières et la nature de l'aliment récolté, nectar ou pollen.

Chaque butineuse a été observée à son retour au nid, et la nature de sa récol-
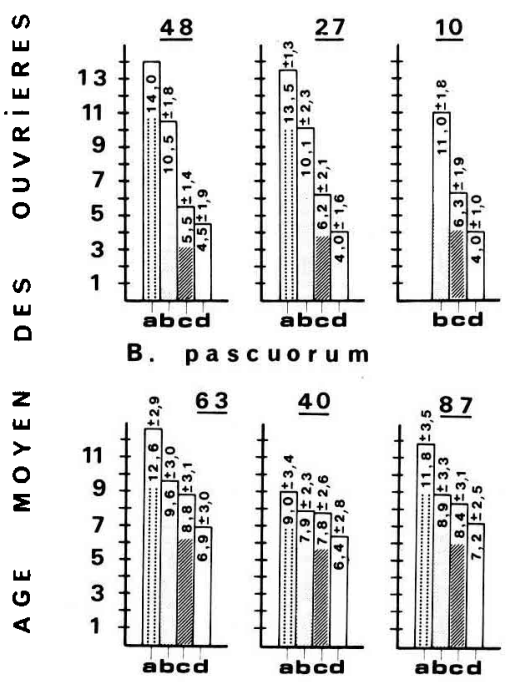

B. I ucorum

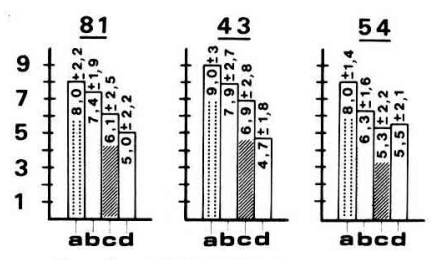

B. I a pidarius

TAILLE DES OUVRIERES

Fig. 3. Age moyen (en jours) des ouvrières de Pyr. (Mel.) lapidarius (colonies 81, 43, 54), de B. lucorum (colonies $63,40,87$ ), et de Meg. (Thor.) pascuorum (colonies 48, 27, 10) à la première sortie en fonction de leur taille. En abscisses : taille des ouvrières de chaque colonie. Critère de taille : longueur de la cellule radiale : a : 2,1-2,3 $\mathrm{mm}$; $\mathrm{b}: 2,4-2,6 \mathrm{~mm}$; $\mathrm{c}$ : 2,7-2,9 mm; d : 3-3,2 mm. En ordonnées : âge moyen (en jours) des ouvrières à la première sortie. 


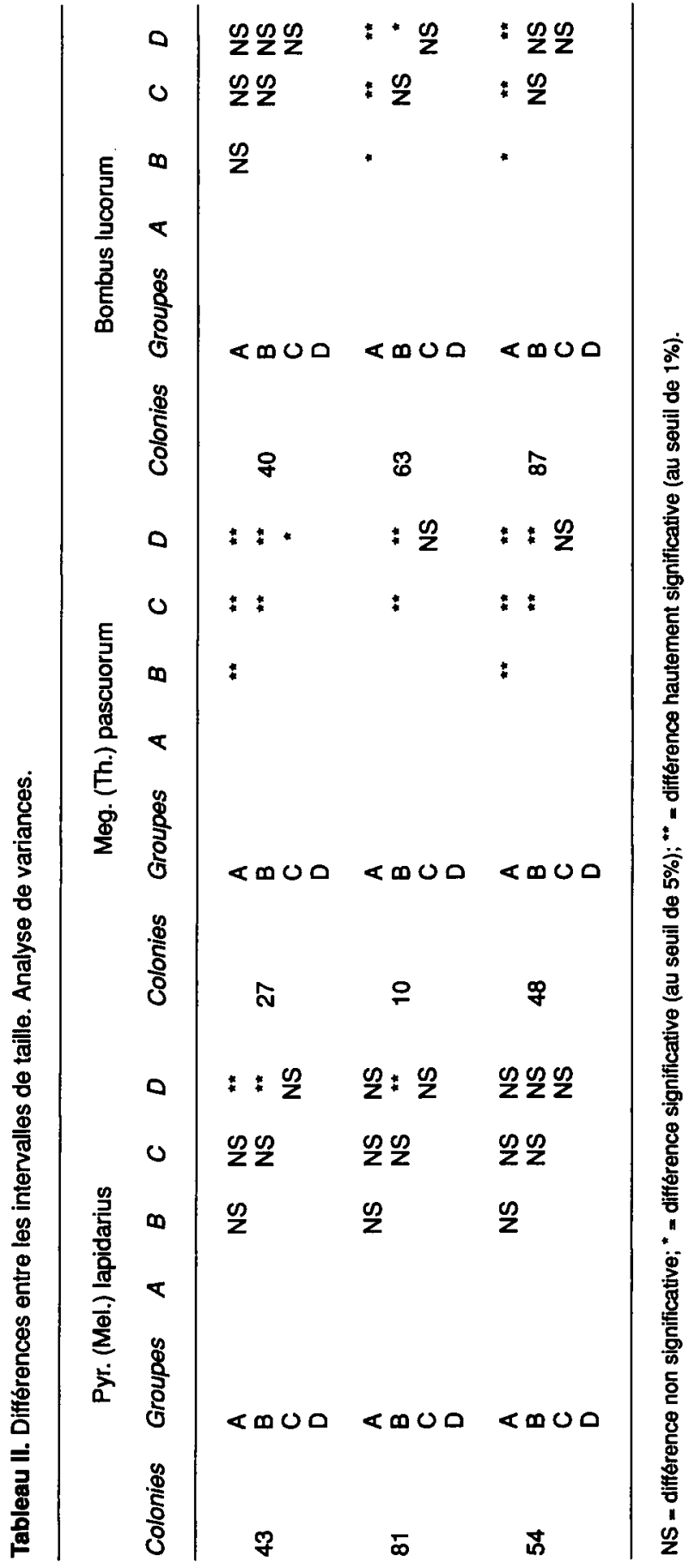


te notée. Le Tableau III rapporte le nombre d'ouvrières ayant récolté soit du nectar, soit du pollen, d'une façon permanente, c'est-à-dire au cours de sorties successives pendant une semaine. Les ouvrières dont la nature de la récolte change pendant ce laps de temps figurent dans la rangée «nectar ou pollen». L'examen de ce tableau fait apparaître :

- un nombre de butineuses constantes $(98,133)$ inférieur à celui des butineuses inconstantes $(114,136)$ respectivement chez $P$. lapidarius et chez $B$. lucorum, alors que les valeurs sont inversées chez M. pascuorum (94 contre 81, respectivement), dans le cadre de nos observations; - un nombre de butineuses de nectar $(48,65)$ inférieur à celui des butineuses de pollen $(50,68)$ respectivement chez les 2 espèces $P$. lapidarius et $B$. lucorum. $M$. pascuorum présente des valeurs inverses : respectivement 50 et 44.

Si l'on effectue des comparaisons, intervalle de taille par intervalle de taille, entre les "butineuses constantes" de nectar et celles de pollen, nous constatons que les butineuses de petite taille (intervalles de taille $A$ et $B$ ) sont plus nombreuses à récolter du nectar que du pollen, à l'exception de $B$. lucorum, où les 2 catégories de butineuses présentent le même nombre (28). La récolte du pollen est effectuée par un plus grand nombre de butineuses de grande taille (intervalles de taille $C$ et $D$ ) chez les 3 espèces.

Tebleau III. Nombre et pourcentage d'ouvrières butineuses de nectar ou de pollen en fonction de leur taille chez Pyr. (Mel.) lapidarius, Meg. (Thor.) pascuorum et Bombus lucorum.

\begin{tabular}{|c|c|c|c|c|c|c|}
\hline \multirow[t]{2}{*}{$\begin{array}{l}\text { Nature de } \\
\text { l'aliment récolté }\end{array}$} & \multirow[t]{2}{*}{ Espèce } & \multicolumn{5}{|c|}{$\begin{array}{l}\text { Nombre (et pourcentage) d'ouvrières } \\
\text { en fonction de leur taille (1) }\end{array}$} \\
\hline & & $A$ & $B$ & $c$ & $D$ & Total \\
\hline Nectar & $\begin{array}{l}\text { P. lapidarius } \\
\text { M. pascuorum } \\
\text { B. lucorum }\end{array}$ & $\begin{array}{c}4 \\
(8,3 \%) \\
2 \\
(4,0 \%) \\
6 \\
(9,2 \%)\end{array}$ & $\begin{array}{c}24 \\
(50,0 \%) \\
25 \\
(50,0 \%) \\
22 \\
(33,8 \%)\end{array}$ & $\begin{array}{c}18 \\
(37,5 \%) \\
21 \\
(42,0 \%) \\
27 \\
(41,5 \%)\end{array}$ & $\begin{array}{c}2 \\
(4,2 \%) \\
2 \\
(4,0 \%) \\
10 \\
(15,4 \%)\end{array}$ & $\begin{array}{l}50 \\
65\end{array}$ \\
\hline Pollen & $\begin{array}{l}\text { P. lapidarius } \\
\text { M. pascuorum } \\
\text { B. lucorum }\end{array}$ & $\begin{array}{c}2 \\
(4,0 \%) \\
1 \\
(2,3 \%) \\
5 \\
(7,4 \%)\end{array}$ & $\begin{array}{c}20 \\
(40,0 \%) \\
12 \\
(27,3 \%) \\
23 \\
(33,8 \%)\end{array}$ & $\begin{array}{c}24 \\
(48,0 \%) \\
27 \\
(61,4 \%) \\
29 \\
(42,6 \%)\end{array}$ & $\begin{array}{c}4 \\
(8,0 \%) \\
4 \\
(9,1 \%) \\
11 \\
(16,2 \%)\end{array}$ & $\begin{array}{l}50 \\
44\end{array}$ \\
\hline $\begin{array}{l}\text { Nectar } \\
\text { ou pollen }\end{array}$ & $\begin{array}{l}\text { P. lapidarius } \\
\text { M. pascuorum } \\
\text { B. lucorum }\end{array}$ & $\begin{array}{c}6 \\
(5,3 \%) \\
2 \\
(2,5 \%) \\
13 \\
(9,6 \%)\end{array}$ & $\begin{array}{c}52 \\
(45,6 \%) \\
40 \\
(49,4 \%) \\
43 \\
(31,6 \%)\end{array}$ & $\begin{array}{c}46 \\
(40,3 \%) \\
31 \\
(38,3 \%) \\
56 \\
(41,2 \%)\end{array}$ & $\begin{array}{c}10 \\
(8,8 \%) \\
8 \\
(9,9 \%) \\
24 \\
(17,6 \%)\end{array}$ & $\begin{array}{r}81 \\
136\end{array}$ \\
\hline
\end{tabular}

(1) : longueur de la celiule radiale : A : 2,1-2,3 mm; B : 2,4-2,6 mm; C :2,7-2,9 mm; D : 3-3,2 mm. 


\section{Discussion}

En suivant l'évolution individuelle des ouvrières de chaque colonie chez 3 espèces de Bombus, nous avons pu constater que la totalité des ouvrières sont d'abord nourrices. Certaines le demeurent toute leur vie, mais la plupart changent de spécialité, devenant des butineuses.

L'activité des ouvrières nourrices se manifeste par des soins au couvain, le nettoyage et la construction des cellules cireuses, la régulation de la température du nid.

L'échelonnement des premières sorties d'ouvrières, sur une période de 2 à 15 jours, montre que les activités liées au milieu extérieur s'effectuent de manière progressive jusqu'au $7^{e}$ ou $8^{e}$ jour chez Bombus lucorum et $P$. lapidarius : c'est l'âge moyen à partir duquel la majorité des ouvrières effectuent leur première sortie chez ces 2 espèces.

Deux maximums apparaissent chez M. pascuorum, à des intervalles de 7 à 8 jours. L'observation des ouvrières à leur première sortie a permis de regrouper celles-ci en 2 catégories, distinctes par la taille : les ouvrières les plus précoces sont plus grosses que celles dont la première sortie est plus tardive.

$\mathrm{Ce}$ polymorphisme fait intervenir un facteur épigénétique : la taille des ouvrières adultes issues d'une même couvée est liée à la quantité de nourriture reçue pendant le développement larvaire.

La répartition temporelle du travail permet aux jeunes ouvrières de Bombus de rester dans le nid, alors que les ouvrières plus âgées butinent. Cependant, le lien entre le travail exécuté et l'âge de l'insecte n'est pas strict.

Nous avons constaté que chaque ouvrière ne passe pas forcément par toutes les étapes du cycle chronologique des tâches sociales sans en omettre aucune. Une séquence d'activité peut être écourtée, répétée ou retardée selon les circonstances.

L'âge auquel commencent les activités de butinage chez $M$. pascuorum n'est pas modifié par l'enlèvement des butineuses de la colonie ou par de courtes périodes d'inanition (Brian, 1952, 1954). Cet auteur conclut que le changement de comportement est le résultat de modifications ontogéniques liées à des différences de poids. La modification du comportement se réalise plus précocement chez les ouvrières de grande taille que chez les petites ouvrières. Nos résultats corroborent les observations de Brian (1952) sur M. pascuorum; cet auteur, en prenant comme critère biométrique le poids, a montré que les ouvrières les plus lourdes commençaient à butiner à environ 5 jours, la plupart des autres à 15 jours, tandis que certaines, parmi les plus légères, ne quittaient jamais le nid. Selon Brian, l'âge auquel une ouvrière de $M$. pascuorum commence à butiner n'a aucun rapport avec les besoins de la colonie; il s'agit d'un caractère constitutionnel, non plastique, qui varie d'un individu à l'autre et se trouve lié à des différences de poids.

Free (1955), de son côté, a constaté que les activités des ouvrières dépendaient des besoins de la colonie. Ainsi, lorsque des butineuses étaient retirées d'une colonie, les plus jeunes ouvrières, normalement nourrices, entreprenaient des travaux de butinage. De même, le retrait d'ouvrières nourrices entraînait un certain nombre de butineuses à s'acquitter de tâches au sein du nid. Le retrait des butineuses n'a entraîné aucune modification d'activités, selon Brian, alors que Free a constaté une régulation. L'éthogenèse est liée, d'après Brian, à des facteurs endogènes. Mais les différences d'interprétation tiennent plutôt à la durée 
du retrait : 24 heures pour Brian, quelques jours pour Free.

Selon Kugler (1943), le retrait du miel des pots du nid entraîne une augmentation de l'activité de butinage, mais ne modifie pas l'âge auquel les ouvrières effectuent leur première sortie.

Cette interchangeabilité des ouvrières a été également constatée par Sakagami et Zucchi (1965). Chaque ouvrière peut entreprendre n'importe quelle activité, et, lorsque les butineuses se retrouvent dans le nid, elles s'occupent d'،activités ménagères". Toutefois, nous avons pu observer qu'un certain nombre de butineuses repartent dès qu'elles se sont débarrassées de leurs provisions dans les pots à miel ou dans les poches à pollen. D'autres semblent marquer une pause.

L'aptitude des ouvrières d'une colonie de $B$. terrestris à maintenir, après enlèvement de la moitié d'entre elles, le niveau de l'alimentation des larves, montre que ces insectes sont capables d'ajuster leur comportement social, en déployant une activité plus intense, en réponse aux besoins de la colonie (Pendrel et Plowright, 1981). La performance de l'ensemble de la colonie constituerait un effet de masse résultant d'opérations en partie stochastiques des ouvrières. Les facteurs responsables de cet ajustement ne sont pas identifiés. Ces auteurs ajoutent que les ouvrières ne peuvent pas être réparties en "nourrices" et «non nourrices" dans ces conditions expérimentales.

Le seul comportement au sein du nid imputable à une ouvrière d'âge connu correspond à un fonctionnement de certaines glandes en relation avec l'alimentation des larves et la construction du nid. Les glandes hypopharyngiennes et les glandes cirières des ouvrières sont fonctionnelles dès le $2^{\theta}$ jour de leur vie imaginale. Les glandes hypopharyngiennes se développent jusqu'au $5^{e}$ jour, puis régres- sent généralement à partir du $10^{\circ}$. Toutefois, en l'absence de jeunes ouvrières pour prendre en charge l'élevage des larves, l'activité de ces glandes peut se prolonger jusqu'au $50^{\circ}$ jour. La période de sécrétion des glandes cirières dure jusqu'au $10^{\circ}$ jour environ (Röseler, 1967).

Ce phénomène de prolongation du comportement de nourrice a été démontré chez l'abeille domestique (Milojevic, 1940; Haydak, 1963). L'absence de jeunes ouvrières entraînent les nourrices plus âgées à s'occuper de l'élevage du couvain et à maintenir leurs glandes hypopharyngiennes fonctionnelles. Cependant, les ouvrières issues du couvain élevé par les "vieilles" nourrices présentent un poids plus faible et une durée de vie plus courte que celles élevées par des jeunes nourrices. L'absence d'ouvrières constructrices chez l'abeille peut provoquer un nouveau développement des glandes cirières chez les butineuses (Örösi-Pal, 1956). De même, les glandes hypopharyngiennes des butineuses régénèrent lorsque le nombre des jeunes ouvrières diminue brusquement. Une telle régénération au niveau des glandes des butineuses n'a pu encore être nettement démontrée chez les Bourdons.

Le diminution progressive de l'âge moyen auquel les ouvrières de chaque espèce effectuent leur première sortie peut s'expliquer, en partie, par la présence de larves, dont le nombre croissant entraîne une augmentation de l'intensité de la recherche de nourriture, et, par conséquent, une accélération du début de l'activité des butineuses.

Cette action stimulante exercée par le couvain sur les ouvrières se retrouve chez différentes espèces de Fourmis (revue in Lenoir, 1979) et chez Vespa orientalis $F$. (Vespidae) (Ishay et Landau, 1972). La présence de couvain non operculé chez Apis mellifera stimule la récolte de pollen 
(Free, 1967), constituant nécessaire de la nourriture larvaire, et influe sur la division temporelle du travail en incitant les ouvrières à devenir des butineuses à un âge plus précoce (Winston et Fergusson, 1985).

II apparaît que la taille des ouvrières intervient parmi les facteurs de la spécialisation du travail chez les bourdons. Ce polyéthisme de caste a été observé par un certain nombre d'auteurs (Coville, 1890; Sladen, 1912; Meidell, 1934; Jordan, 1936; Richards, 1946), mais ce sont surtout les travaux de Cumber (1949), Brian (1952), Free (1955), Miyamoto (1959), Sakagami et Zucchi (1965) qui ont cherché à expliquer les corrélations entre la taille, ou le poids, et le comportement des ouvrières.

Free et Butler (1959) ont suggéré qu'une telle division du travail basée sur la taille pouvait présenter 2 avantages : les petites ouvrières sont plus habiles à se mouvoir à travers les passages étroits et complexes du nid que les grosses ouvrières; les grosses ouvrières, qui passent une grande partie de leur temps à butiner, peuvent récolter davantage de nectar et de pollen, et sont plus résistantes en vol lorsque les conditions météorologiques sont défavorables.

Les grosses ouvrières sont plus aptes, selon Heinrich (1979), à assurer la régulation de leur température corporelle et à visiter les fleurs à corolle profonde que les petites. Chez Bombus terricola, les grandes ouvrières seraient capables de parcourir des distances plus longues pour rapporter des charges en nectar et en pollen plus lourdes (Morse, 1978). Cet auteur a observé également que lorsque la densité des populations de bourdons était élevée, en conditions naturelles, les petites butineuses cherchaient à éviter la présence des grandes, ce qui réduisait quelque peu leurs possibilités de récolte.

Si les petites ouvrières semblent être plus souvent «nourrices» et les grandes, "butineuses", ce schéma n'est pas applicable de manière aussi stéréotypée à chaque espèce de Bombus. D'après nos résultats, la taille revêt une importance plus grande dans la distribution des activités entre nourrices et butineuses chez $M$. pascuorum que chez $B$. lucorum et $P$. lapidarius. Cette relation entre la taille et le type d'activité est plus élevée chez pascuorum, autrement dit chez une espèce où le polymorphisme est plus marqué que chez lucorum et lapidarius. Les observations de Valenzuela-Gonzalez (1981) vont également dans ce sens. Le polymorphisme observé chez les ouvrières est plus net chez les espèces "pocket-making"* (M. pascuorum) que chez les espèces "pollen-storing" ( $B$. lucorum, P. lapidarius).

Chez Bombus (F.) morio, un certain nombre d'ouvrières ne quittent jamais le nid, mais leur taille n'est pas pour autant la plus petite; certaines atteignent une taille identique ou supérieure à celle des butineuses (Garofalo, 1978).

Les plus grosses ouvrières tendent à récolter relativement plus de nourriture que les petites. Les capacités de transport plus importantes de la nourriture liquide chez les ouvrières de plus grande taille pourraient être imputables aux possibilités d'emmagasinage plus grandes du jabot, la

" Cette distinction repose sur le mode d'approvisionnement des larves :

- chez les espèces du premier groupe, les larves se nourrissent du pollen accumulé dans de petites poches de cire accolées à la chambre larvaire;

- chez les espéces "pollen-storing", les larves se nourrissent directement de pollen au début de leur vie, puis sont alimentées, par régurgitation, par la reine ou les ouvrières à travers un orifice de l'enveloppe cireuse de la chambre larvaire. 
dilatation de celui-ci serait la conséquence d'une spécialisation dans l'activité de butinage. Une telle caractéristique anatomique a été mise en évidence chez Lasius niger (Lenoir, 1979).

Chez les bourdons, nos résultats démontrent (Tableau IV) que, en général, les butineuses de petite taille récoltent du nectar plutôt que du pollen; chez les individus de grande taille, on constate le processus inverse. Une butineuse de bourdon peut transporter, au cours d'un voyage, environ $50 \%$ du poids de son propre corps en nectar, tandis que la charge de pollen correspond en moyenne à $20 \%$. Cette différence dans le poids relatif des charges se trouve modérée par le tait que les butineuses de nectar ne récoltent que cet aliment, alors que les butineuses de pollen rapportent généralement en plus une petite provision de nectar. II s'établit ainsi, dans une certaine mesure, une répartition du travail parmi les butineuses. Nos observations corroborent celles de Brian (1952), de Free (1955) et de Miyamoto (1957). Chez $M$. pascuorum, $26 \%$ des butineuses, dont le poids était supérieur à $150 \mathrm{mg}$, retournaient au nid sans charge de pollen, alors que chez les butineuses d'un poids inférieur à $150 \mathrm{mg}, 72 \%$ rentraient sans pelotes de pollen (Brian).

Des phénomènes analogues se retrouvent chez d'autres insectes sociaux. Chez Paravespula vulgaris L. (Hymenoptera, Vespidae), Archer (1977) a trouvé une corrélation entre la taille des individus et la quantité de liquide rapportée au nid. Chez les Formicidae, la corrélation "tailletype d'activité" est plus marquée chez les espèces à polymorphisme fort (Camponotus sp.) que chez celles à polymorphisme faible (Formica sp., Solenopsis sp.) (Lenoir, 1979).

Les quantités de nectar et de pollen emmagasinées dans les colonies d'abeilles domestiques peuvent influencer les activités de butinage (Free, 1965); il serait intéressant d'étudier les effets de ce facteur sur la répartition du travail dans les colonies de bourdons.

\section{Conclusion}

Chez les bourdons, le polymorphisme social revêt un double aspect : ontogénique et statural. Dans le premier cas, la colonie de bourdons présenterait quelques similitudes avec les sociétés d'abeilles domestiques et de certaines espèces de Meliponini (Sommeijer, 1984), chez lesquelles le polyéthisme est lié fondamentalement au facteur "âge", mais on observe des différences individuelles plus ou moins importantes.

La délimitation des classes d'âge en accord avec des comportements déterminés présente, chez les bourdons, une incertitude. L'âge auquel intervient un changement de comportement est variable, suivant l'espèce considérée, les colonies de la même espèce, et même le numéro de la couvée. Des inversions dans l'exécution d'une tâche peuvent se produire, en rapport avec les besoins de la colonie, autrement dit l'état des réserves alimentaires.

Si l'appartenance à une classe d'âge ne suffit pas à conditionner un comportement déterminé, la catégorie morphologique d'une ouvrière de bourdon peut expliquer en partie la modification de son comportement. D'après nos observations, c'est chez les espèces à polymorphisme marqué, telles que $M$. pascuorum, que la taille apparaît plus nettement en rapport avec une fonction, nourrice ou butineuse. Chez les espèces où le polymorphisme des ouvrières est moins accusé ( $B$. lucorum, $P$. lapidarius), la relation entre la taille et la fonction est plus incertaine. 
Toutes les tâches sont susceptibles d'être effectuées par une ouvrière de bourdon au cours de sa vie. Mais la période d'exécution de ces tâches, leur succession et leur réversibilité éventuelle présentent des variations plus ou moins importantes qui peuvent s'expliquer, en partie, par les besoins de la colonie, en particulier du couvain. Notre étude a été effectuée en considérant essentiellement le comportement alimentaire. Un prolongement de ce travail devrait viser à étudier la nature des ressources externes et l'importance des réserves alimentaires du nid, afin de déterminer la répartition temporelle du travail et la coordination des activités des ouvrières d'une colonie. De récents travaux (Robinson et Page, 1988; Frumhoff et Baker, 1988) ont montré qu'il existait dans les colonies d'abeilles domestiques une variation génétique en liaison avec des différences dans l'accomplissement des tâches. Chez un certain nombre d'espèces de bourdons, qui se caractérisent par une polyandrie, il conviendrait d'aborder certains problèmes tels que la construction, la défense du nid, les comportements de soin au couvain, et de compléter ainsi nos connaissances sur les differents aspects de la répartition du travail chez ces insectes.

\section{Remerciements}

Je remercie M.R. Coutin, M.R. Darchen et les lecteurs anonymes pour leurs suggestions et leurs critiques dans la rédaction de ce texte.

\section{Références}

Archer M.E. (1977) The weights of forager loads of Paravespula vulgaris L. (Hymenoptera, Vespidae) and the relationships of load weight to forager size. Insectes Soc. 24, 95102

Brian A.D. (1952) Division of labour and foraging in Bombus agrorum Fab. J. Anim. Ecol. 21 (2), 223-240

Brian A.D. (1954) The foraging of BumbleBees. Part I. Foraging behaviour. Bee World 35 (4), 61-67

Coville F.V. (1890) Notes on bumble-bees. Proc. Entomol. Soc. Wash. 1 (4), 197-202

Cumber R.A. (1949) The biology of BumbleBees, with special reference to the production of the worker caste. Trans. R. Entomol. Soc. Lond. 100, 1-45

Free J.B. (1955) The division of labour within Bumblebee colonies. Insectes Soc. 2, 195-212

Free J.B. (1965) The allocation of duties among worker honeybees. Symp. Zool. Soc. Lond. 14, 39-59

Free J.B. (1967) Factors determining the collection of pollen by honeybee foragers. Anim. Behav. 15, 134-144

Free J.B. \& Butler C.G. (1959) Bumblebees. Collins, London

Frumhoff P.C. \& Baker J. (1988) A genetic component to division of labour within honey bee colonies. Nature 333, 358-361

Garofalo C.A. (1978) Bionomics of Bombus (Fenvidobombus) morio. 2. Body size and length of life of workers. J. Apic. Res. 17, 130-136

Haydak M.H. (1963) Age of nurse bees and brood rearing. J. Apic. Res. 2, 101-103

Heinrich B. (1979) Bumblebee Economics. Harvard University Press, Cambridge (Mass.)

Ishay J. \& Landau E.M. (1972) Vespa larvae send out rythmic hunger signals. Nature 237, 286-287

Jordan R. (1936) Beobachtungen der Arbeitsteilung im Hummelstaate (Bombus muscorum). Arch. Bienenkd. 17, 81-91

Kugler H. (1943) Hummeln als Blütenbesucher. Ergeb. Biol. 19, 143-323

Lenoir A. (1979) Le comportement alimentaire et la division du travail chez la Fourmi Lasius niger. Bull. Biol. Fr. Belg. CXIII, 79-314

Meidell O. (1934) Fra dagliglivet $i$ et homlebol. Naturen 58, 85-95 et 108-116

Michener C.D. (1974) The Social Behavior of the Boes. A Comparative Study. Belknap Press of Harvard University Press, Cambridge (Mass.) 
Milojevic B.D. (1940) A new interpretation of the social life of the honey bee. Bee World 21, 39-41

Miyamoto S. (1957) Behavior study on Bombus ardens Smith in early stage of nesting. Sci. Rep. Hyogo Univ. Agric. 3 (Ser. Agric. Biol.) 1-5

Miyamoto S. (1959) On the individuality in the behavior of workers of Bombus ardens Smith and Bombus diversus Smith. Akitu 8, 35-36 (in Japanse)

Morse D.H. (1977) Estimating proboscis length from wing length in Bumble-bees (Bombus sp.) Ann. Entomol. Soc. Am. 70, 311-315

Morse D.H. (1978) Foraging rate, foraging position and worker size in Bumble-Bee workers. Proc. IVth Intern. Symp. on Pollination. Md. Agric. Exp. Sta. Spec. Misc. Publ. 1, 447452

Nowogrodzki R. (1984) Division of labour in the honeybee colony : a review. Bee world 65, 109-116

Örösi-Pal Z. (1956) AZ épitö alkalom hatasa a viaszmirigy müködéséne. Méhészet, Budap. 4, 105

Pendrel B.A. \& Plowright R.C. (1981) Larval feeding by adult Bumble-bee workers (Hymenoptera : Apidae). Behav. Ecol. Sociobiol. 8, 71-76

Pouvreau A. \& Marilleau R. (1979) L'élevage des bourdons. Leur utilisation pour la pollinisation des plantes. Cah. liaison OPIE, nos 24-26

Richards O.W. (1946) Observations on Bombus agrorum Fab. (Hymenoptera, Bombidae). Proc. R. Entomol. Soc. Lond. 21, 66-71
Robinson G.E. \& Page R.E. Jr. (1988) Genetic determination of guarding and undertaking in honey-bee colonies. Nature 333, 356-358

Rösch G.A. (1930) Untersuchungen über die Arbeitsteilung im Bienenstaat. 2. Teil. Die Tätigkeiten der Arbeitsbienen unter experimentell veränderten Bedingungen. Z. Vgl. Physiol. 12, 1-71

Róseler P.F. (1967) Arbeitsteilung und Drüsenzustände in Hummelvölkern. Naturwissenschaften 54, 146-147

Sakagami S.G. \& Zucchi R. (1965) Winterverhalten einer neotropischen Hummel, Bombus atratus, innerhalb des Beobachtungskastens. Ein Beitrag zur Biologie der Hummeln. $J$. Fac. Sci. Hokkaido Univ. (VI, Zool.) 15, 712-762 Sladen F.W.L. (1912) The Humble-Bee, Its Life History and How to Domesticate it. London, McMillan and Co.

Sommeijer M.J. (1984) Distribution of labour among workers of Melipona favosa F.; agepolyethism and worker oviposition. Insectes Soc. 31, 171-184

Valenzuela-Gonzalez J.E. (1981) Contribucion al estudio de la division del trabajo en los abejorros : Bombus pascuorum Scop. y Bombus lapidarius L. Folia Entomol. Mex. 49, 121-139

Weir J.S. (1958) Polyethism in workers of the Ant Myrmica. Insectes Soc. 5, 97-128 et 315339

Wilson E.O. (1971) The Insect Societies. Harvard University Press, Cambridge (Mass.)

Winston M.L. \& Fergusson L.A. (1985) The effect of worker loss on temporal caste structure in colonies of the honeybee (Apis mellifera L.) Can. J. Zool. 63, 777-780 